\title{
Temporal order memory of the rat in spontaneous object recognition: effects of number of items, exposure interval, and retention time
}

\author{
Taichi Hatakeyama, Manami Sugita, Kazuo Yamada, and Yukio Ichitani \\ Institute of Psychology and Behavioral Neuroscience, University of Tsukuba, Tsukuba, Ibaraki 305-8577, Japan
}

\begin{abstract}
Temporal order memory was analyzed using a spontaneous object recognition (SOR) paradigm in rats. In SOR, animals were allowed to explore freely two or five different objects sequentially in the sample phase, and then, two different objects shown in the sample phase were simultaneously presented, and exploration time of object shown earlier compared with that shown later was regarded as a discrimination index. Here we investigated the effects of (1) number of sample items, (2) sample exposure interval, and (3) retention time between the sample and test phases on temporal order memory in SOR. In experiment 1, rats showed significant discrimination between the objects presented earlier and later under five-item, but not two-item, condition. In experiment 2, using the five-item condition, longer retention time (between sample and test phases) induced lower discrimination and longer sample exposure interval produced higher discrimination. In experiment 3 , using the two-item condition, longer sample exposure interval produced higher discrimination and longer retention time tended to make rats' discrimination worse. Taken together, these results indicate that rats' performance in this test paradigm was better with more sample items and longer exposure intervals as well as shorter retention time, suggesting that the familiarity to items and the temporal distance from the present to the occurrence of events affect temporal order memory.
\end{abstract}

Temporal order memory is defined as memory for the order in which items/events have been experienced (Hannesson et al. 2004). It also includes another important form of memory, which in its simplest application supports the ability to discriminate the relative recency of stimuli/events, i.e., how long ago an item was encountered (Barker and Warburton 2011a), although we cannot differentiate between temporal order memory and recency or familiarity discrimination in most studies on this topic. It has been shown that normal rats can integrate "what," "where," and "when" information to remember the order of a sequence of events (Ergorul and Eichenbaum 2004). However, whether nonhuman animals are able to consciously recollect past experiences and whether they have a sense of time are still under debate: episodiclike memory in rats was based on how long ago it occurred and not on when (Roberts et al. 2008), while there is evidence for remembering when an event occurred that does not depend on the judgments of familiarity (Zhou and Crystal 2009; Crystal 2018).

In rodents, previous studies have investigated temporal order memory using spatial locations in a radial maze (Kesner and Novak 1982; Chiba et al. 1994; Sugita et al. 2013), odor stimuli (Fortin et al. 2002), or spontaneous explorations for objects (Mitchell and Laiacona 1998; Hunsaker et al. 2008; Barker and Warburton 2011b; Tam et al. 2014; Barker et al. 2017). In temporal order memory tasks using spatial locations or odor stimuli, rats encountered five to eight different spatial locations or odor stimuli sequentially in the study phase, and then, two different familiar items of the study phase were simultaneously presented. The animals had to choose the item presented earlier, which required extensive training and the use of reinforcement. In the task using spontaneous explorations for objects, animals were allowed to explore freely two to four different objects sequentially in the sample phase, and then, two different familiar items of the sample phase were simul-

Corresponding author: y-ichit@human.tsukuba.ac.jp

Article is online at http://www.learnmem.org/cgi/doi/10.1101//m.048215. 118. taneously presented. The animals tended to explore old familiar than recent familiar objects, although any task trainings and the use of reinforcement were not required. Thus, use of spontaneous explorations for objects is beneficial since it avoids confounds that could be introduced by using other tasks described above.

It is thought that successful performance of temporal order memory may depend on three factors: (1) number of items in the sample/study phase, (2) interval of each sample exposure in the sample/study phase, (3) retention time between the sample/study and test phases. In previous studies using a radial maze, it was suggested that the number of items presented in the sequence was critical for successful temporal order memory, but not the interval length between items in the study phase (Sugita et al. 2013). On the other hand, in spontaneous object explorations, it was suggested that the sample exposure interval, as well as the retention time, was important for successful temporal order memory (Mitchell and Laiacona 1998; Tam et al. 2014). However, the involvement of number of items in temporal order memory still remains unclear. Furthermore, in humans using temporal order judgment with naturalistic stimuli such as movies or video clips, temporal order judgment retrieval times were negatively correlated with the temporal separation between events in the movie (Kwok and Macaluso 2015), suggesting that larger sample exposure interval makes human subjects easier to remember temporal order of event stimuli. However, in rats using spontaneous object explorations there has been no direct investigation on the relationship between effects of sample exposure interval and retention time.

Thus, in the present study using spontaneous object recognition (SOR) paradigm, first we investigated the effect of number of items in the sample phase (two or five items) on the temporal order

(C) 2018 Hatakeyama et al. This article is distributed exclusively by Cold Spring Harbor Laboratory Press for the first 12 months after the full-issue publication date (see http://learnmem.cshlp.org/site/misc/terms.xhtml). After 12 months, it is available under a Creative Commons License (Attribution-NonCommercial 4.0 International), as described at http://creativecommons.org/licenses/by-nc/ 4.0/. 
discrimination in experiment (Exp.) 1. In the five-item condition of this experiment, the first and fifth presentation stimuli in the sample phase were not used in the test phase to exclude the possibility that primacy and recency effects could affect performance. Second, we investigated the effects of retention time and sample exposure interval on temporal order discrimination using five items (Exp. 2) and two items (Exp. 3). Taking these considerations into account, we discussed the involvement of number of items, inter-exposure interval in the sample phase, and retention time in temporal order memory performance.

Male Wistar-Imamichi rats $(n=60)$ were used as subjects. They were housed individually and kept on a $12 \mathrm{~h}$ light-dark cycle with continuous access to food and water. All behavioral testing was conducted during the light phase (08:00-20:00). Animal experiments were approved by the University of Tsukuba Committee on Animal Research.

Temporal order memory test using the SOR was conducted in an open-field arena $(90 \times 90 \times 45 \mathrm{~cm})$ made of polyvinylchloride. The walls of the arena were colored in black, while the floor was gray. An overhead camera and a video recorder were used to monitor and record the animals' behavior for the analysis. The illumination of the center of the arena was $65 \mathrm{~lx}$. The stimulus objects were copies of 15 different objects made of glass, metal, or plastic and varied in height between 9 and $15 \mathrm{~cm}$. All the objects were heavy enough or fixed on the heavy metal plate so that the rats could not move them during testing.

Prior to the SOR test, rats received $5 \mathrm{~min}$ of handling and were habituated to the arena without any objects for $10 \mathrm{~min}$ for three consecutive days. The SOR test consisted of a sample phase (two or five exposures), retention time, and a test phase (see Fig. 1). For instance, in the first exposure of the sample phase, a pair of

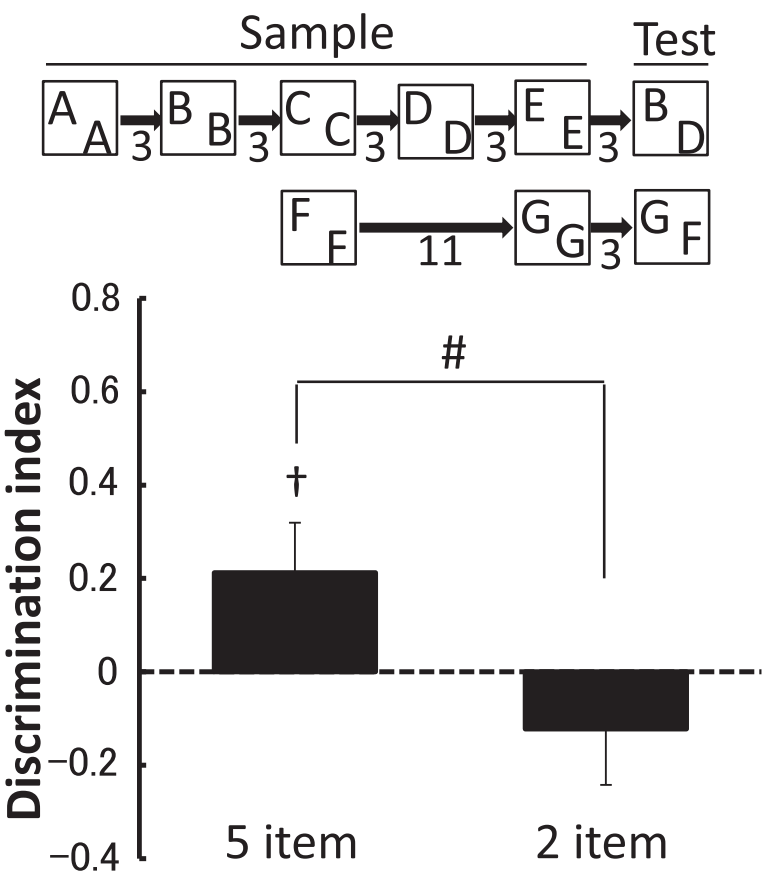

Figure 1. Effects of number of items on the discrimination performance in the test phase (Exp. 1). Schematic drawings of the SOR test and the results are shown. Capital letters in squares represent objects, and numbers between squares show sample exposure interval (min). Rats explored different objects for 5 min each $(A \rightarrow B \rightarrow C \rightarrow D \rightarrow E$, or $F \rightarrow G)$. Then, after the retention time of $3 \mathrm{~min}$, the test phase $(5 \mathrm{~min})$ was conducted ( $B$ versus $D$ or $F$ versus $G$ ). Mean discrimination indexes \pm SEM are shown. $(\dagger) P<0.1$ versus chance (0). (\#) $P<0.05$. identical objects was placed diagonally in the arena (the center of each object was $22.5 \mathrm{~cm}$ from adjacent two walls), and the rat was released in the center of the arena. Each rat was allowed to explore freely these objects for $5 \mathrm{~min}$. After the exposure, the rat was removed and returned to its home cage. In the second exposure, another pair of identical objects was presented. This procedure was repeated two or five times, and then after the retention time, the test phase started, in which two objects were placed at the same positions as in the sample phase. One object was the old object that was exposed earlier in the sample phase, and the other was the recent object that was exposed recently. The positions of the old/recent objects in the test phase were counterbalanced. The animal was placed into the arena and allowed to explore the objects for $5 \mathrm{~min}$.

In both the sample and test phases, we measured time rats explored the objects. Exploration was defined as the rat directing its nose toward the object within a distance of $2 \mathrm{~cm}$. Touching the object with other parts of the body or climbing over the object was not included. The analysis was conducted on the first 2 min of the test phase. As a measure of discrimination, "discrimination index (DI)" was calculated by dividing the difference in exploration time between the two objects (old object - recent object) by the total amount of exploration for two objects (old object + recent object). The arena was wiped with a wet cloth containing sodium hypochlorite solution and the objects were cleaned up with $70 \%$ ethanol to eliminate odor cues.

In Exp. 1, effects of number of items were investigated (Fig. 1). Different pairs of objects were presented in each sample exposure. The inter-exposure interval was $3 \mathrm{~min}$ in five-sample exposure condition, and $11 \mathrm{~min}$ in two-sample exposure condition. After the retention time of $3 \mathrm{~min}$, the test phase started. In the fivesample exposure condition, rats were exposed to the second and fourth objects, while in the two-sample exposure condition, they were exposed to the first and second objects. Twelve rats were used, and they experienced both two- and five-sample exposure conditions with an interval of $2 \mathrm{~d}$. The order of experiences was counterbalanced.

In Exp. 2, using five items effects of retention time and sample exposure interval were investigated. First, we investigated how long rats can retain temporal order memory using five items (Fig. 2A: Exp. 2-1). The sample phase consisted of five sample exposures with different objects, and the sample exposure interval was $3 \mathrm{~min}$. After the retention time of 3,10, or $30 \mathrm{~min}$, the test phase started, in which the second and fourth objects were presented. Twelve rats experienced all retention times with an interval of at least $2 \mathrm{~d}$, and they were counterbalanced with the order of experiences. Second, using the retention time (10 min) in which rats could not retain the temporal order memory in Exp. 2-1, we investigated the effects of sample exposure interval (Fig. 2B: Exp. 2-2). The procedure was the same as in Exp. 2-1 except that the sample exposure interval was 3,10 , or $30 \mathrm{~min}$ and the retention time was fixed with $10 \mathrm{~min}$. Twelve rats experienced all sample exposure interval conditions with an interval of at least $2 \mathrm{~d}$. The order of conditions was counterbalanced.

In Exp. 3, using two items effects of sample exposure interval and retention time were tested. First, we investigated whether rats can discriminate the temporal order of two items when longer intervals than $11 \mathrm{~min}$ were inserted between exposures (Fig. 2D: Exp. 3-1). In Exp. 2-2 we confirmed that, with a longer sample exposure interval (30 $\mathrm{min}$ ), rats could explore the old (second) object more than the recent (fourth) object even after longer retention time of $10 \mathrm{~min}$. Thus, here we investigated the effects of sample exposure interval on temporal order memory in SOR with two items. The sample phase consisted of two sample exposures with different objects. The sample exposure interval was 11,65 , or $125 \mathrm{~min}$. After the retention time of $3 \mathrm{~min}$, the test phase started, in which the 
A

Exp. 2-1: Effects of retention time (5 items)
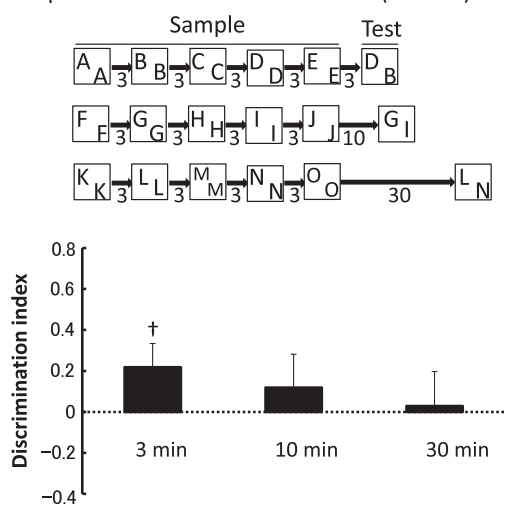

C

Exp. 3-2: Effects of retention time (2 items)
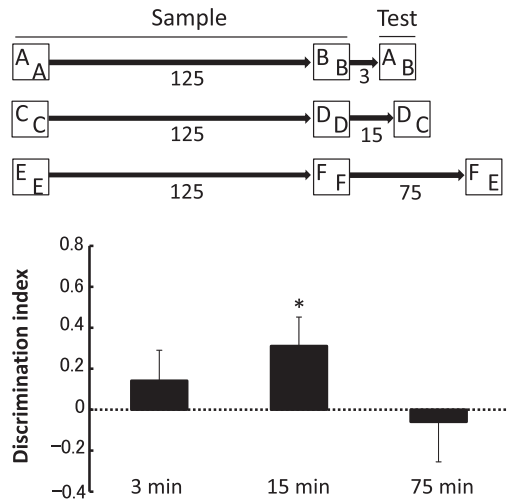

B

Exp. 2-2: Effects of sample exposure interval (5 items)
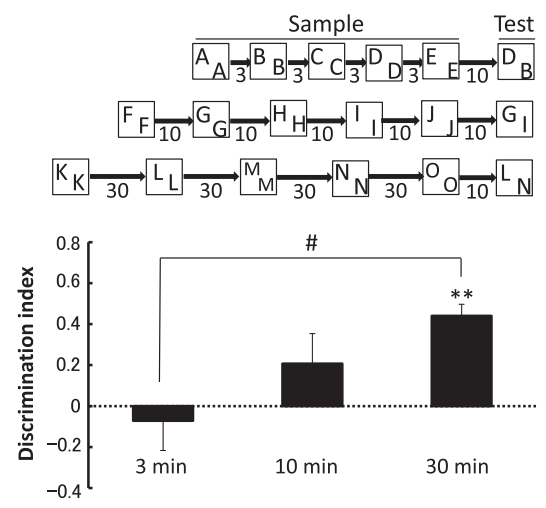

D

Exp. 3-1: Effects of sample exposure interval (2 items)

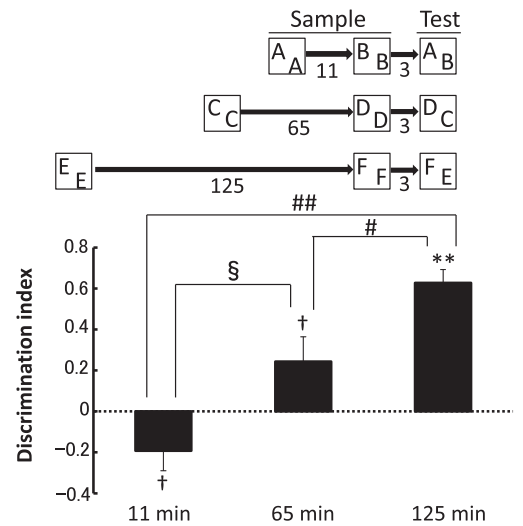

Figure 2. Effects of retention time and sample exposure interval on the discrimination performance in Exp. $2(A, B)$ and Exp. $3(C, D)$. Schematic drawings of the SOR test and the results are shown. Capital letters in squares represent objects, and numbers between squares show sample exposure interval (min). (A) Effects of retention time in Exp. 2-1. Rats explored different objects $(A \rightarrow B \rightarrow C \rightarrow D \rightarrow E, F \rightarrow G \rightarrow H \rightarrow$ $\mathrm{I} \rightarrow \mathrm{J}$, or $\mathrm{K} \rightarrow \mathrm{L} \rightarrow \mathrm{M} \rightarrow \mathrm{N} \rightarrow \mathrm{O}$ ). Then, after the retention times of 3, 10, or 30 min, the test phase started ( $B$ versus $D, G$ versus $I$, or $L$ versus $N$ ). Mean discrimination indexes (DI) $\pm S E M$ in three retention time conditions are shown. $(\dagger) P<0.1$ above chance $(0)$. (B) Effects of sample exposure interval in Exp. 2-2. Rats explored different objects $(A \rightarrow B \rightarrow C \rightarrow D \rightarrow E, F \rightarrow G \rightarrow H \rightarrow I \rightarrow I$, or $K \rightarrow L \rightarrow M \rightarrow N \rightarrow O)$, and the sample exposure intervals were 3,10 , or $30 \mathrm{~min}$. Then, after the retention time of $10 \mathrm{~min}$, the test phase was conducted ( $B$ versus D, G versus I, or L versus N). Mean DIs \pm SEM in three inter-exposure interval conditions are shown. (**) $P<0.01$ versus chance (0). (\#) $P<0.05$. (C) Effects of retention time in Exp. 3-2. Rats explored different objects $(A \rightarrow B, C \rightarrow D$, or $E \rightarrow F)$. Then, after the retention time of 3, 15 , or $75 \mathrm{~min}$, the test phase was conducted (A versus $B, C$ versus D, or E versus $F$ ). Mean DIs \pm SEM in three retention time conditions are shown. $\left({ }^{*}\right) P<0.05$ versus chance $(0)$. (D) Effects of sample exposure interval in Exp. 3-1. Rats explored different objects $(A \rightarrow B, C \rightarrow D$, or $E \rightarrow F)$, and the sample exposure intervals were 11,65 , or $125 \mathrm{~min}$. Then, after the retention time of $3 \mathrm{~min}$, the test phase was conducted (A versus $B, C$ versus $D$, or E versus $F$ ). Mean DIs \pm SEM in three inter-exposure interval conditions are shown. $\left({ }^{* *}\right) P<0.01$ versus chance $(0)$. ( $\dagger$ ) $P<0.1$ versus chance $(0)$. ( ()$P<0.1$. (\#) $P<0.05$. (\#\#) $P<0.01$.

first and second objects were placed. Twelve rats experienced all sample exposure interval conditions with an interval of at least $2 \mathrm{~d}$. The order of conditions was counterbalanced. Second, we investigated how long rats can retain the temporal order memory using two items (Fig. 2C: Exp. 3-2). The procedure was the same as in Exp. 3-1, except that the sample exposure interval was fixed with $125 \mathrm{~min}$ and the retention time was 3, 15, or $75 \mathrm{~min}$. Twelve rats experienced all retention times with an interval of at least $2 \mathrm{~d}$. The order of experiences was counterbalanced.

In the sample phases of all experiments, exploration time of sample objects in each sample exposure $(5 \mathrm{~min})$ and statistical results are shown in Table 1.

In Exp. 1, mean DIs of five- and two-item conditions in the test phase are shown in Figure 1. DI of five-item condition was higher than that of two-item. A paired $t$-test revealed that there was a significant difference $\left(t_{(11)}=2.25, P<0.05\right)$. In comparison with expected chance level of DI (0), DI of five-item condition tended to be higher than chance $\left(t_{(11)}=1.99, P<\right.$ $0.10)$ and DI of two items was not significantly below zero.

In Exp. 2-1, mean DIs of three retention time conditions in the test phase are shown in Figure 2A. A one-way repeated analysis of variance (ANOVA) showed no main effect. In comparison with chance level (0), DI of $3 \mathrm{~min}$ condition tended to be higher than chance $\left(t_{(11)}=\right.$ $1.88, P<0.10$ ), but not 10 and $30 \mathrm{~min}$ conditions. In Exp. 2-2, mean DIs of three inter-exposure interval conditions in the test phase are shown in Figure 2B. A one-way repeated ANOVA showed a significant main effect $\left(F_{(2,22)}=3.68, P<\right.$ $0.05)$. A post hoc Bonferroni test revealed that DI of 30 min condition was significantly higher than that of $3 \mathrm{~min}(P<$ $0.05)$. In comparison with chance level (0), DI of 30 min condition was higher than chance $\left(t_{(11)}=7.70, P<0.01\right)$, but not 3 and 10 min conditions.

In Exp. 3-1, mean DIs of three interexposure interval conditions in the test phase are shown in Figure 2D. A one-way repeated ANOVA showed a significant main effect $\left(F_{(2,22)}=19.52, P<0.01\right)$. A post hoc Bonferroni test revealed that DI of 125 min condition was higher than those of $11 \mathrm{~min}(P<0.01)$ and $65 \mathrm{~min}$ conditions $(P<0.05)$, and DI of $65 \mathrm{~min}$ condition tended to be higher than that of $11 \mathrm{~min}(P<0.10)$. In comparison with chance level (0), DI of 125 min condition was higher than chance $\left(t_{(11)}=9.63, P<\right.$ $0.01)$. Also, DI of 65 min condition tended to be higher than chance level $\left(t_{(11)}=\right.$ $2.00, P<0.10)$, but that of 11 min condition tended to be lower than chance $\left(t_{(11)}=1.95, P<0.10\right)$. In Exp. 3-2, mean DIs of three retention time conditions in the test phase are shown in Figure 2C. A one-way repeated ANOVA showed that there was not a significant main effect. In comparison with chance level (0), DI of 15 min condition was higher than chance $\left(t_{(11)}=2.21, P<0.05\right)$, but not in the $3 \mathrm{~min}$ and $75 \mathrm{~min}$ conditions.

The present study investigated the effects of (1) number of sample items, (2) sample exposure interval, and (3) retention time between the sample and test phases on rats' temporal order memory in the SOR. Experiment 1 investigated the effect of number of sample items on temporal order memory, showing that the performance in five-sample condition was better than that in twosample condition. Using the five-sample condition of Exp.1, Exp. 2 investigated the effects of retention time and sample exposure interval, and showed that the performance became worse as the retention time was extended (Exp. 2-1), but with longer sample exposure intervals, the performance improved even after the retention time of $10 \mathrm{~min}$ (Exp. 2-2). Using the two-sample condition of 
Temporal order memory tested by object recognition

Table 1. Exploration time (sec) of sample objects in each sample exposure (5 min) of Exp. 1, 2, and 3

\begin{tabular}{|c|c|c|c|c|c|c|c|c|c|c|c|}
\hline \multirow{3}{*}{$\begin{array}{l}\text { Experiment } \\
\text { Exp. 1: effect of number of items }(\mathrm{RT}=3 \mathrm{~min})\end{array}$} & \multirow{3}{*}{$\begin{array}{l}\text { Condition } \\
5 \text { items } \\
2 \text { items }\end{array}$} & \multicolumn{10}{|c|}{ Exposure } \\
\hline & & \multicolumn{2}{|c|}{$1 \mathrm{st}$} & \multicolumn{2}{|c|}{ 2nd } & \multicolumn{2}{|c|}{$3 r d$} & \multicolumn{2}{|c|}{ 4th } & \multicolumn{2}{|c|}{ 5th } \\
\hline & & $\begin{array}{l}53.9 \\
33.5\end{array}$ & $\begin{array}{r}(10.5) \\
(4.3)\end{array}$ & $\begin{array}{l}53.9 \\
44.3\end{array}$ & $\begin{array}{l}(6.8) \\
(6.1)\end{array}$ & 58.1 & $(10.2)$ & 47.5 & $(8.2)$ & 50.1 & $(9.8)$ \\
\hline Exp. 2-1: effect of RT (5 items; IEI = $3 \mathrm{~min}$ ) & $\begin{array}{l}3 \mathrm{~min} \\
10 \mathrm{~min} \\
30 \mathrm{~min} \\
3 \mathrm{~min} \\
10 \mathrm{~min} \\
30 \mathrm{~min}\end{array}$ & $\begin{array}{l}40.2 \\
55.9 \\
57.9 \\
60.5 \\
51.8 \\
47.4\end{array}$ & $\begin{array}{l}(5.9) \\
(7.9) \\
(6.9) \\
(7.9) \\
(5.5) \\
(9.2)\end{array}$ & $\begin{array}{l}57.7 \\
46.4 \\
42.4 \\
41.0 \\
52.7 \\
54.0\end{array}$ & $\begin{array}{l}(7.6) \\
(4.2) \\
(4.7) \\
(5.5) \\
(7.5) \\
(7.8)\end{array}$ & $\begin{array}{l}72.1 \\
70.8 \\
76.0 \\
56.2 \\
59.1 \\
77.9\end{array}$ & $\begin{array}{r}(8.6) \\
(13.7) \\
(5.6) \\
(5.9) \\
(4.6) \\
(6.4)\end{array}$ & $\begin{array}{l}43.9 \\
56.5 \\
43.9 \\
50.0 \\
52.7 \\
53.1\end{array}$ & $\begin{array}{r}(6.1) \\
(11.4) \\
(6.4) \\
(8.0) \\
(7.2) \\
(7.3)\end{array}$ & $\begin{array}{l}49.7 \\
29.5 \\
54.3 \\
56.7 \\
70.7 \\
57.1\end{array}$ & $\begin{array}{r}(10.6) \\
(5.5) \\
(7.4) \\
(7.2) \\
(9.8) \\
(8.3)\end{array}$ \\
\hline Exp. 3-2: effect of RT (2 items; IEI = $125 \mathrm{~min}$ ) & $\begin{array}{l}11 \mathrm{~min} \\
65 \mathrm{~min} \\
125 \mathrm{~min} \\
3 \mathrm{~min} \\
15 \mathrm{~min} \\
75 \mathrm{~min}\end{array}$ & $\begin{array}{l}58.1 \\
59.7 \\
55.4 \\
46.9 \\
59.0 \\
50.1\end{array}$ & $\begin{array}{l}(5.2) \\
(6.6) \\
(6.4) \\
(6.5) \\
(7.8) \\
(5.4)\end{array}$ & $\begin{array}{l}70.8 \\
58.0 \\
59.9 \\
36.7 \\
56.4 \\
50.1\end{array}$ & $\begin{array}{l}(8.6) \\
(8.3) \\
(5.9) \\
(7.5) \\
(6.5) \\
(8.1)\end{array}$ & & & & & & \\
\hline
\end{tabular}

Means $( \pm$ SEM) are shown. IEl, inter-exposure interval in the sample phase; RT, retention time. In Exp. 1, in the five-item condition there was no statistical difference of exploration time among five sample exposures according to a one-way analysis of variance (ANOVA) with repeated measures. Similarly, in the two-item condition there was no difference of the exploration time between two sample exposures according to a paired $t$-test. In Exp. $2-1$, according to a two-way repeated ANOVA, there was a main effect of exposure $\left(F_{(4,44)}=7.41, P<0.01\right)$, as well as a significant interaction between RT and exposure $\left(F_{(8,88)}=2.39, P<0.05\right)$. However, a post hoc Bonferroni test revealed that there was not a significant difference of the exploration time between the second and fourth sample objects that were used in the test phase. The main effect of RT was not significant. In Exp. 2-2, according to a two-way repeated ANOVA, there was a main effect of exposure $\left(F_{(4,44)}=5.03, P<0.01\right)$. The main effect of IEl and the interaction between the two factors were not significant. A post hoc Bonferroni test showed that there was not a significant difference of exploration time between the second and fourth sample exposures. In Exp. 3, according to a two-way repeated ANOVA, there were no significant main effects of IEl, sample exposures, and the interaction between the two factors (Exp. 3-1). Additionally, in Exp. 3-2 there was no significant difference of the exploration time among RT conditions and among sample exposures. The interaction between the two factors was not found.

Exp. 1, Exp. 3 investigated the effects of sample exposure interval and retention time, and showed that the performance became better as the sample exposure intervals was extended (Exp. 3-1), but with longer retention time, the performance was deteriorated even when the sample exposure interval was long enough (125 $\min$, Exp. 3-2).

In the present study, it was shown that maintaining the temporal order memory performance was easier in the five-sample item condition than in two-sample item condition (Exp. 1). This finding supports Sugita et al. (2013), but there are two differences of main procedures between these two studies in comparing the effect of number of sample items. First, in the test phase of fivesample item group, Sugita et al. (2013) used two items that were presented first and last in the study phase. It can be speculated that these two items strongly reflected both the primacy and recency effects. In the present study, on the other hand, to reduce these effects, the test phase of five-sample item condition used two items that were exposed second and fourth in the sample phase. Second, the present study used the SOR task to avoid confounds that could be introduced by using a task in which spatial information processing, reference memory, and hunger motivation are required. Thus, it seems that the behavior tested in the present study reflects purer temporal order memory.

The difference of performance depending on the number of sample items can be interpreted according to an assumption of familiarity contrast by Sugita et al. (2013) in which they investigated the effect of number of study phase items using the temporal order memory task in a radial maze. Both of two items presented in the test phase were familiar to rats because they had already experienced these items in the sample phase. In the SOR test of the present study, however, the rats' familiarity to the items was thought to decrease when other items followed in the sample phase. That is, the more items were presented after the second object in the sample phase, the weaker the familiarity with it in the sequence became. On the other hand, the familiarity with the item presented fourth in the sequence did not seem to change so much regardless of the number of items in the sample phase, since rats experienced only one more object before the test phase had been conducted. Therefore, difference of familiarity between the two items seems to be larger in the five-item condition than in the two-item condition. If rats use this familiarity's difference between two items when they encounter two objects simultaneously, this could explain why the temporal order discrimination was higher when a larger number of items were presented in the sample phase. Thus, in the present study, the difference of familiarity between the five-sample item condition in which the second and fourth were shown and the two-sample item condition in which the first and second were shown may have affected the temporal order memory performance. In order to conclude this idea, further experiments in which times from the occurrence of the old or recent items to its retrieval are controlled will be needed.

We found that in both the five- and two-sample item conditions, the temporal order memory performance was worse as the retention time was extended (Exp. 2-1 and 3-2). This finding supports previous studies (Kesner and Novak 1982; Mitchell and Laiacona 1998). For example, Mitchell and Laiacona (1998) using a temporal order memory task of SOR showed that rats could retain the temporal order memory for $24 \mathrm{~h}$, but not more than $72 \mathrm{~h}$. In another report (Kesner and Novak 1982) temporal order memory performance in a radial maze with retention time of $20 \mathrm{sec}$ was better than that with $10 \mathrm{~min}$, although it was dependent on the paired stimuli used for the test phase. Thus, regardless of the number of sample items or the difference of task procedures, the retention time generally affects the temporal order memory.

The present study showed the temporal order memory performance in both the five- and two-sample item conditions was improved with longer sample exposure intervals (Exp. 2-2 and 3-1). This finding agrees with Tam et al. (2014) using the SOR in which extended interval length between the first and second sample items improved temporal order memory. However, Sugita et al. (2013) demonstrated using a radial maze that the interval length 
between items in the study phase did not affect the acquisition of temporal order memory. The discrepancy of the effect of the sample exposure interval between Sugita et al. (2013) and the present study may reflect distinct mechanisms for temporal order memory in these two tests used. Sugita et al. (2013) demonstrated the temporal order discrimination performance was not improved even after the interval length between items in the study phase was extended, suggesting that the memory strength for each item was not used by rats to perform the task. Additionally, in Fortin et al. (2002) using odor stimuli, the hippocampal lesions did affect remembering the sequential ordering, but did not affect recognizing odors that previously occurred, indicating that different memory strength for recent and earlier items did not necessarily support the temporal order discrimination. Therefore, it can be speculated that specifically in the SOR the sample exposure interval is a critical factor that affects the performance, and that rats may use the difference of memory strength in judging temporal order in the SOR.

Experiments 2 and 3 in the present study showed that the sample exposure interval and retention time affect temporal order memory performance. This can be interpreted from the scale-invariant memory, perception, and learning (SIMPLE) model by Brown et al. (2007). According to this model, (1) traces of items are represented in memory partly in terms of their temporal distance from the present, (2) similar mechanisms govern retrieval from memory over many different timescales, and (3) performance on a range of memory tasks is determined by interference from near psychological neighbors. For example, two different memory traces encoded five versus $25 \mathrm{sec}$ in the past will be as confusable with each other as two traces encoded five versus $25 \mathrm{~min}$ in the past (5:25 for both cases). The actual temporal distances are magni-
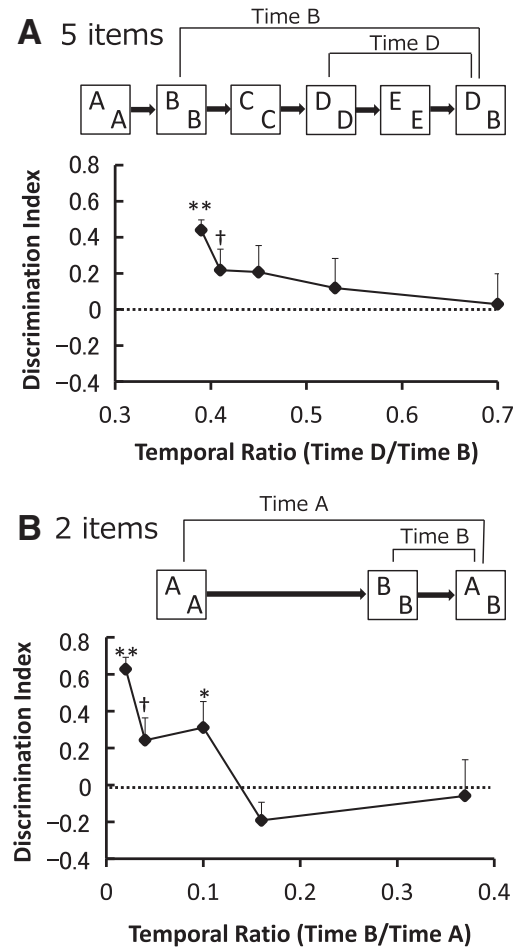

Figure 3. Effects of temporal ratios on DI in the SOR. Schematic drawings of the SOR test and the results obtained in each condition are shown. Capital letters in squares represent objects. Temporal ratios were calculated by dividing Time $D$ by Time $B$ in five-item condition $(A)$, and Time $B$ by Time $A$ in two-item condition $(B)$, respectively. $\left({ }^{* *}\right) P<0.01$, $\left({ }^{*}\right) P<0.05$, and $(\dagger) P<0.10$ versus chance $(0)$. Means \pm SEM are shown. fied by 60 times, but the scale of similarity between the two events in question is kept constant (Kwok and Macaluso 2015). In humans, the authors investigated whether a scale invariance pattern might exist in retrieving the temporal order of events from episodic memory, demonstrating that the performance required for order judgment deteriorated with increasing temporal similarity between episodic events, and that this relationship held across a wide range of temporal distances between events and retention intervals between encoding and retrieval. In agreement with their study, the present study showed that the shorter the sample exposure interval, the more difficult the temporal order memory performance would be. Furthermore, using the data of Exp. 2 and 3 in the present study, temporal ratio was calculated by dividing the temporal distance from the occurrence to its retrieval (recent item) by the other temporal distance (old item) (Fig. 3). It seems that DI decreases as a function of temporal ratio value, which means that discrimination between two different events depends on their temporal distance from the present to the occurrence of them. In terms of a range of retention times, the present study used a narrow range (3-75 $\mathrm{min})$ but their study used a wider range $(0.5 \mathrm{sec}-24 \mathrm{~h})$. Thus, further studies need to extend the retention time to examine whether a scale invariance might exist in retrieving the temporal order memory in the SOR.

The present study revealed that the number of sample items, sample exposure interval between items, and retention time are important factors in temporal order memory in the SOR. Results suggest that (1) a larger number of sample items exposed improve the temporal order memory, (2) the longer retention time, the worse the temporal order memory becomes, (3) with longer sample exposure interval, the temporal order memory is improved. Research of the relationship between the retention time and sample exposure interval may help us further understand the mechanism underlying temporal order memory in the SOR.

\section{Acknowledgments}

This study was supported in part by grants from Japan Society for the Promotion of Science (16K04419, 15K04180).

\section{References}

Barker GRI, Warburton EC. 2011a. Evaluating the neural basis of temporal order memory for visual stimuli in the rat. Eur J Neurosci 33: 705-716.

Barker GRI, Warburton EC. 2011b. When is the hippocampus involved in recognition memory? J Neurosci 31: 10721-10731.

Barker GRI, Banks PJ, Scott H, Ralph GS, Mitrophanous KA, Wong LF, Bashir ZI, Uney JB, Warburton EC. 2017. Separate elements of episodic memory subserved by distinct hippocampal-prefrontal connections. Nat Neurosci 20: 242-250.

Brown GDA, Neath I, Chater N. 2007. A temporal ratio model of memory. Psychol Rev 114: 539-576.

Chiba AA, Kesner RP, Reynolds AM. 1994. Memory for spatial locations as a function of temporal lag in rats: role of hippocampus and medial prefrontal cortex. Behav Neural Biol 61: 123-131.

Crystal JD. 2018. Animal models of episodic memory. Comp Cogn Behav Rev 13: $105-122$.

Ergorul C, Eichenbaum H. 2004. The hippocampus and memory for "what," "where," and "when." Learn Mem 11: 397-405.

Fortin NJ, Agster KL, Eichenbaum HB. 2002. Critical role of the hippocampus in memory for sequences of events. Nat Neurosci 5: $458-462$.

Hannesson DK, Howland JG, Phillips AG. 2004. Interaction between perirhinal and medial prefrontal cortex is required for temporal order but not recognition memory for objects in rats. J Neurosci 24: 4596-4604.

Hunsaker MR, Fieldsted PM, Rosenberg JS, Kesner RP. 2008. Dissociating the roles of dorsal and ventral CA1 for the temporal processing of spatial locations, visual objects, and odors. Behav Neurosci 122: 643-650.

Kesner RP, Novak JM. 1982. Serial position curve in rats: role of the dorsal hippocampus. Science 218: 173-175. 
Kwok SC, Macaluso E. 2015. Scale invariance of temporal order discrimination using complex, naturalistic events. Cognition 140: 111-121.

Mitchell JB, Laiacona J. 1998. The medial frontal cortex and temporal memory: tests using spontaneous exploratory behavior in the rat. Behav Brain Res 97: 107-113.

Roberts WA, Feeney MC, MacPherson K, Petter M, McMillan N, Musolino E. 2008. Episodic-like memory in rats: is it based on when or how long ago? Science 320: $113-115$.

Sugita M, Yamada K, Ichitani Y. 2013. Effects of number of items and interval length on the acquisition of temporal order discrimination in radial maze in rats. Learn Mem 20: 125-129.
Tam SKE, Robinson J, Jennings DJ, Bonardi C. 2014. Dissociations in the effect of delay on object recognition: evidence for an associative model of recognition memory. J Exp Psychol Anim Learn Cogn 40: 106-115.

Zhou W, Crystal JD. 2009. Evidence for remembering when events occurred in a rodent model of episodic memory. Proc Natl Acad Sci 106: 9525-9529.

Received July 16, 2018; accepted in revised form September 4, 2018. 


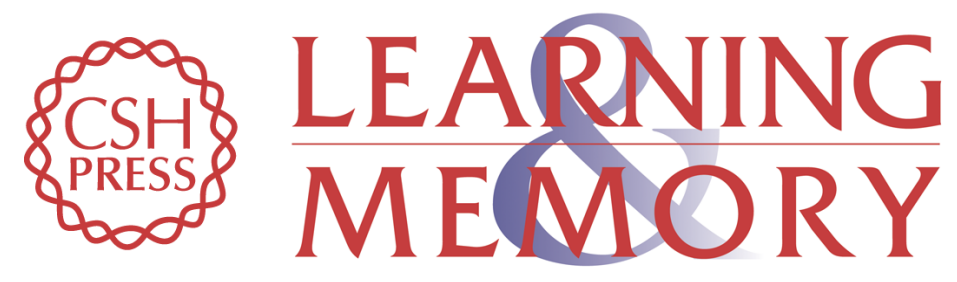

\section{Temporal order memory of the rat in spontaneous object recognition: effects of number of items, exposure interval, and retention time}

Taichi Hatakeyama, Manami Sugita, Kazuo Yamada, et al.

Learn. Mem. 2018, 25:

Access the most recent version at doi:10.1101//m.048215.118

References This article cites 17 articles, 7 of which can be accessed free at: http://learnmem.cshlp.org/content/25/11/574.full.htmI\#ref-list-1

Creative This article is distributed exclusively by Cold Spring Harbor Laboratory Press for the Commons first 12 months after the full-issue publication date (see

License http://learnmem.cshlp.org/site/misc/terms.xhtml). After 12 months, it is available under a Creative Commons License (Attribution-NonCommercial 4.0 International), as described at http://creativecommons.org/licenses/by-nc/4.0/.

Email Alerting Receive free email alerts when new articles cite this article - sign up in the box at the Service top right corner of the article or click here. 